\title{
Failure to Capture with Acceptable Safety Margin
}

National Cancer Institute

\section{Source}

National Cancer Institute. Failure to Capture with Acceptable Safety Margin. NCI

Thesaurus. Code C99953.

Failure to capture manifests as a high pacing threshold that results in either intermittent failure to capture at maximal programmed output or excessive battery drain leading to premature battery exhaustion. (ACC) 